\title{
Breakdown characteristics and optically visible discharge paths of surface flashover
}

\begin{abstract}
Characteristics of surface flashover in the presence of different dielectric materials have been investigated. Lightning voltage impulses $(1.2 / 50 \mathrm{\varepsilon s})$ have been applied to a rod-plane gap of $250 \mathrm{~mm}$, bridged by cylindrical solid dielectrics with circular cross-section of $10 \mathrm{~mm}$. The materials used are polythene, polymethyl methacrylate (PMMA), and polyvinyl chloride (PVC). The characteristics of breakdown are compared with those pertinent to a similar rodplane air gap. In each case, $50 \%$ breakdown voltage was found for both polarities. Then for each material 10 impulses were applied at 100\% breakdown voltage for each polarity. The results show that there are three different types of paths taken by the discharges; they are the discharges that leap out of the rod at the HV triple junction (type-A), those follow the surface for a certain length and then leap out of the surface (type-B) and those entirely follow the surface (type-C).
\end{abstract}

Keyword: Lightning impulse; Surface flashover; Optical path; Breakdown strength; Insulation 